In IS4TM: International Symposium on Surgery Simulation and Soft Tissue Modelling Lecture Notes in Computer Science (LNCS 2673), N. Ayache and H. Delingette (Eds), Springer Verlag, New York. 2003. pp. 311-320.

\title{
Haptic Simulation of a Tool In Contact With a Nonlinear Deformable Body
}

\author{
Mohsen Mahvash $^{1}$ and Vincent Hayward ${ }^{2}$ \\ 1 Real Contact Inc. \\ Lorne Crescent, Montréal, Québec, Canada H2X 2B1 \\ 2 Center for Intelligent Machines \\ McGill University, Montréal, Québec, Canada, H3A 2A7 \\ \{mahvash, hayward\}@cim.mcgill.ca
}

\begin{abstract}
This paper presents a method to artificially re-create haptic feedback while moving and sliding an arbitrary virtual tool against a virtual deformable body with nonlinear elastic properties. The computation of the response in such general cases is a task which does not yet admit computational solutions suitable for realtime implementation. To address this, we describe an approach based on the bookkeeping of forcedeflections curves stored at the nodes of a triangulated body surface. For realism, normal and lateral deformations at each node are represented in a range of deflection distances. The response everywhere is synthesized via area interpolation of response curves stored at the nodes of the mesh. The mathematical continuity of the synthetic response is the result of both local coordinates interpolation and of response function interpolation, which previous methods did not account for. This guarantees the absence of haptic 'clicks' and 'pops' which are unacceptable artifacts in high fidelity simulations. Sliding contacts are also considered.
\end{abstract}

\section{Introduction}

A haptic display is capable of re-creating artificially the forces that simulate the interaction of a tool with a deformable body. This capability can be used for various applications such as in training for surgical tasks performed with a tool.

The computational engine of a haptic display, namely the haptic engine, should be able to provide these forces in real time. The construction of a haptic engine that can simulate the continuous mechanics of the contact between a tool and a nonlinear deformable body in realtime is a daunting task. Instead, we introduce a pre-calculation approach. It relies on the offline determination of force-deflection curves at the nodes of a triangulated body surface mesh for a range of deflection in a pre-processing step. Online, forces are calculated at arbitrary locations via area interpolation.

The pre-calculation method approach can be viewed as a systems approach. Contact forces are the outputs of a multi-input multi-output system. The inputs are the position and the orientation of the tool tip, and the internal variables represent the position of the contact point. The behavior of the system is represented by a construction of piecewise polynomial curves designed to approximate 


\section{In IS4TM: International Symposium on Surgery Simulation and Soft Tissue Modelling Lecture Notes in Computer Science (LNCS 2673), N. Ayache and H. Delingette (Eds), Springer Verlag, New York. 2003. pp. 311-320.}

the behavior of an actual deformed body. The coefficients of these polynomials are the system parameters. The systems formulation allows us ensure two properties for the simulation which are also properties of the original physical system, namely, continuity and passivity $[15,14]$. Continuity is provided by the interpolation of the coordinates defined at the surface of virtual body and by the interpolation of the response functions.

The identification of the system parameters is done offline. It is performed by fitting the outputs of the system to actual contact forces obtained through measurement or via accurate offline simulation. The parameters can be stored in standard data file format since at any given time, only a small number of them is needed for simulation.

Section 2 reviews previous work. Section 3 describes a mathematical framework to calculate normal and lateral forces. Section 4 explains how the precalculated responses can be constructed from actual or accurate forces at a set of test points. Section 5 evaluates the interpolation approach through a simple implementation. Section 6 concludes the paper.

\section{Related Work}

Various techniques were proposed to make the haptic simulation of contact interaction possible. We divide them into two groups.

\subsection{Pre-calculation Methods}

Cotin et. al., James and Pai, and Bro-Nielsen introduced the pre-calculation method $[4,11,12,16]$. Linear models, obtained either from the finite element method or from the boundary element method, represent the deformation of a body. A set of algebraic linear relations among the nodal quantities at the free boundary are then derived [12]. The deformation responses given by unit forces (or tractions) applied to each node of the free boundary are calculated during a preprocessing phase. The nodal forces in the contact region are calculated in advance from a reduced-order linear system that directly relates the nodal displacements to forces. A small linear system is derived from the displacement responses at runtime. The deformation response of the boundary due to a displacement constraint imposed by the user is calculated as the superposition of responses of each nodal force in a preset contact region.

Delingette et. al. as well as Astley and Hayward used pre-calculation techniques to compute the contact between a part of a body (peripheral to the interaction point) and the rest of the body in a hybrid body structure $[7,3]$.

Pre-calculation methods are very effective for reducing the computation time of deformation. However, they are applicable only to linear elastic bodies and small displacements. Due to discretization, even for linear elastic deformation, accurate computation of the force deflection response requires a large number of elements to represent a body well. In the contact region, this number can be arbitrarily large, and hence can yield a prohibitively large computational load. 


\section{In IS4TM: International Symposium on Surgery Simulation and Soft Tissue Modelling Lecture Notes in Computer Science (LNCS 2673), N. Ayache and H. Delingette (Eds), Springer Verlag, New York. 2003. pp. 311-320.}

In contrast, the approach introduced in this paper is capable of handling nonlinear deformation (arising either from large deformation or from nonlinear elasticity) due to massive pre-computation of the forces responses in a pre-processing step. Moreover, the pre-computation burden can be reduced by computing forces for a mesh structure coarser than the mesh structure used during the simulation.

\subsection{Time Integration Multi-Resolution Methods}

High order dynamic deformation models represent the deformation of the body. Explicit or semi-explicit time integration are used to solve these models efficiently in realtime. Multi-resolution calculations in space and time are used to refine the calculation in regions of interest.

Astley and Hayward proposed multi-layer finite element mesh for modeling deformation of a viscoelastic body [2]. Multi-rate integration could be used to compute the model. Zhang and Canny used the explicit time integration method for haptic rendering of large deformation [18]. D'Aulignac et al. used implicit integration in a particle-based method [5]. Wu et. al. used explicit integration and adaptive meshing for simulating large deformations of non-Hookean materials [17]. Debunne et. al. used adaptive multi-rate integration with multispatial resolution for simulating tool contact with viscoelastic materials. These approaches essentially simplify either the finite element method or the contact problem to make high rate computation of forces possible [6].

\section{System for Tool Contact Simulation}

Figure 1 defines a haptic engine system for a single tool. The inputs to the system are $x^{1}, x^{2}, x^{3}$, the position components of the virtual tool tip (or the end effector of the device) and $\theta^{1}, \theta^{2}, \theta^{3}$ the orientation components of the tool tip. The internal variables of the system are the position components $c^{1}, c^{2}, c^{3}$ of a contact point $c$. Output forces and torques are functions both of the inputs and of the internal variables. This representation of the haptic engine system assumes a quasi-static contact. It also assumes that the contact area between the tool and the body always contains the tool tip.

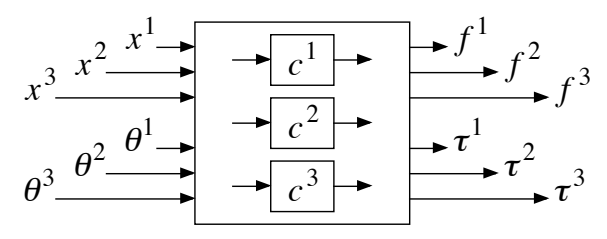

Fig. 1. Haptic engine.

In the following, we describe the calculation of output forces for a haptic engine. A similar approach can be used for the calculation of output torques. We ignore the dependency of contact forces on tool orientation. It could be included following exactly the same approach. 
In IS4TM: International Symposium on Surgery Simulation and Soft Tissue Modelling Lecture Notes in Computer Science (LNCS 2673), N. Ayache and H. Delingette (Eds), Springer Verlag, New York. 2003. pp. 311-320.

\subsection{Normal Contact}

The haptic engine receives $x(t)$, the position of tip of a virtual tool. The contact point $c$ represents the position of the initial contact of the tool on the surface of the deformable body at rest. The deflection vector at point $c$ is $\delta=x-c$, see Figure 2 and caption.
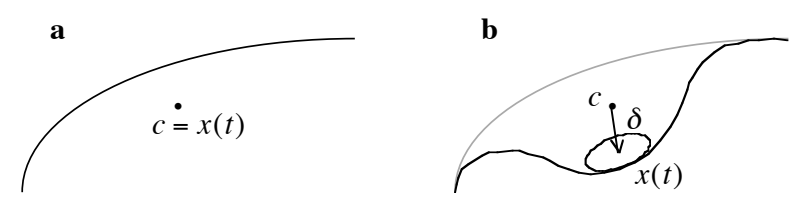

Fig. 2. (a) A tool initially contacts a body at point $c$. (b) With penetration, a contact surface is formed (shown as a circle). The known tool tip position $x$ gives an approximation for the position of this surface. The deflection $\delta$ at point $c$, a point of initial contact on the surface of an undeformed object (in black outline), is found from the difference between the position of the contact surface and $c$. When the object deforms (in black outline), a force response is produced.

Nodes distributed on the surface of the body can be organized in a triangulation used to represent the geometry of the body at rest as a set of triangular elements. With the force response pre-calculation approach, the need to determine the nodal displacements online is eliminated.

Response of a deformable body. The force response to contact at each node $l$ of element $m$ is represented in local coordinates. Each node is labeled $x_{l, m}$. The unit vectors $u_{l, m}^{z}$ and $u_{l, m}^{r}$ with origin at $x_{l, m}$ are such that $\delta_{l, m}=$ $\delta_{l, m}^{z} u_{l, m}^{z}+u_{l, m}^{r} \delta_{l, m}^{r}$, as shown in Figure 3a. The quantities $f_{l, m}^{z}($.$) and f_{l, m}^{r}($. are the components of the force responses as in Figure 3b. The responses can be derived from actual force responses (pre-calculated or pre-measured with suitable approximations [14]) using piecewise polynomial interpolation. Given $x_{l, m}$ and $x$, the force response at node $l$ of element $m$ is:

$$
f_{l, m}(\delta)=f_{l, m}^{r}\left(\delta_{l, m}^{r}\right) u_{l, m}^{r}+f_{l, m}^{z}\left(\delta_{l, m}^{z}\right) u_{l, m}^{z} .
$$
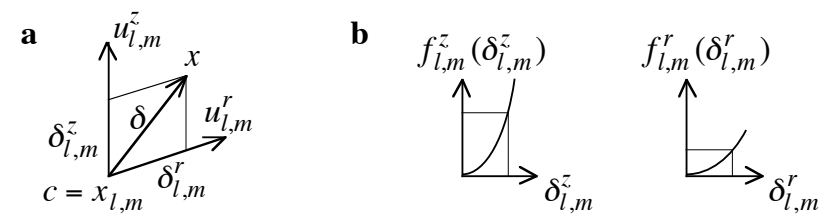

Fig. 3. (a) Deflection in local coordinates. (b) Response vector components. 
In IS4TM: International Symposium on Surgery Simulation and Soft Tissue Modelling Lecture Notes in Computer Science (LNCS 2673),

N. Ayache and H. Delingette (Eds), Springer Verlag, New York. 2003. pp. 311-320.

Interpolated response. When $c$ is inside an element $m$, coordinates are defined at point $c$ with a unit vector $u_{c}^{z}$ found by interpolation of unit vectors at each node.

The unit vector $u_{c}^{r}$ is such that $\delta=\delta_{c}^{z} u_{c}^{z}+\delta_{c}^{r} u_{c}^{r}$ as in Figure 4. Call $n_{l, m}($. the interpolation function for node $l$ of element $m$ :

$$
u_{c}^{z}=\sum_{l=1,2,3} n_{l, m}(c) u_{l, m}^{z}
$$

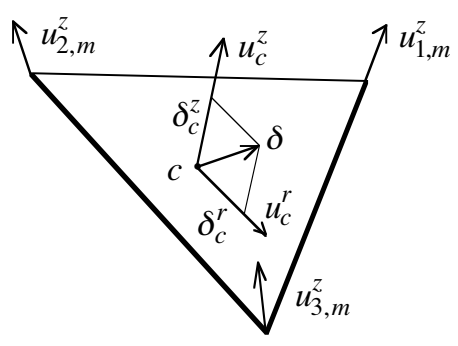

Fig. 4. Local coordinates at $c$. The normal vector $u_{c}^{z}$ points inside the body.

We use a common interpolation scheme used in computational methods for continuous mechanics based on natural coordinates. Within one element of area $A_{m}$, these coordinates are given by the areas $A_{l, m}$ of the triangles defined by the contact point and the three vertices as in Figure 5a,

$$
n_{l, m}(c)=\frac{A_{l, m}(c)}{A_{m}} .
$$

Note that the vector $u_{c}^{z}$ is normally distinct from the vector normal to the surface of the element.

A key property of the interpolation approach is to ensure continuity of coordinate change over the surface of the body. Referring to Figure 5b, the forcedeflection response at $c$ is obtained from:

$$
f_{c}(\delta)=f_{c}^{z}\left(\delta_{c}^{z}\right) u_{c}^{z}+f_{c}^{r}\left(\delta_{c}^{r}\right) u_{c}^{r},
$$

where $f_{c}^{z}($.$) and f_{c}^{r}($.$) are also interpolated from three pre-calculated force-$ deflection responses:

$$
\begin{aligned}
& f_{c}^{z}(.)=\sum_{l=1,2,3} n_{l, m}(c) f_{l, m}^{z}(.), \\
& f_{c}^{r}(.)=\sum_{l=1,2,3} n_{l, m}(c) f_{l, m}^{r}(.) .
\end{aligned}
$$

The haptic engine outputs are now defined for any initial contact point and tool tip position. 
In IS4TM: International Symposium on Surgery Simulation and Soft Tissue Modelling Lecture Notes in Computer Science (LNCS 2673),

N. Ayache and H. Delingette (Eds), Springer Verlag, New York. 2003. pp. 311-320.

$\mathbf{a}$

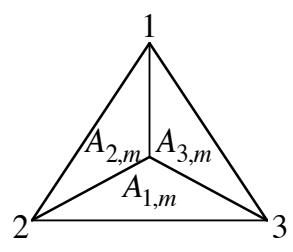

b

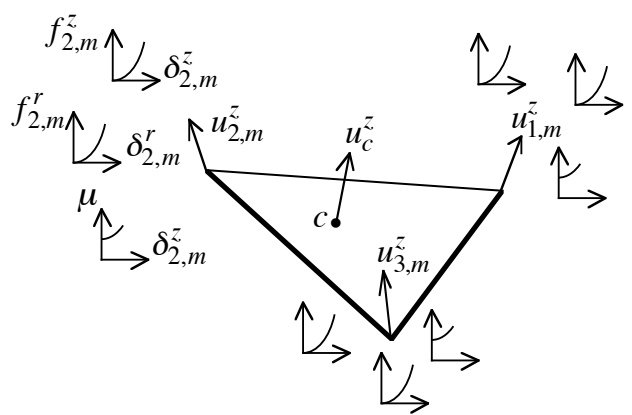

Fig. 5. (a) A point inside a triangle defines three areas labeled as indicated. (b) For each triangular element $m$, the vertices are associated to $u_{l, m}^{z}, l=1,2,3$ and to two responses $f_{l, m}^{z}$ and $f_{l, m}^{r}$. Similarly, variations in the limiting coefficient of friction as a function of penetration can be encoded over the surface of the body.

\subsection{Sliding Contact}

Sliding behavior is represented in terms of pre-sliding displacements [10]. During sliding contact, $c$ moves over the body boundary. Let $\delta_{s}^{r}$ be the limiting sliding deflection such that $f_{c}^{r}\left(\delta_{s}^{r}\right)=\mu\left(\delta_{c}^{z}\right) f_{c}^{z}\left(\delta_{c}^{z}\right)$, where $\mu($.$) is the limiting friction$ coefficient given as function of normal penetration. The pre-sliding distance is not to be confused with the pre-sliding observed with hard materials in contact, which is measured in $\mu \mathrm{m}$ [1]. For soft materials, it can be large and depends mostly on the material properties and on its support. Consider, for example, the case of palpation. The skin can deform over large distances before slip occurs. Applying Coulomb's law is equivalent to assuming that $\mu$ is invariant with the contact surface, i.e., is independent from penetration.

In the pre-sliding model, sliding occurs when $\delta^{r}>\delta_{s}^{r}$ [10]. During sliding, $c$ moves over the body surface in such manner that $\delta^{r} \leq \delta_{s}^{r}$ at all times. The movement is in the direction of the projection of $u_{c}^{r}$ over the element surface and its magnitude is $\left|\delta_{c}^{r}-\delta_{s}^{r}\right|$, see Figure 6 .

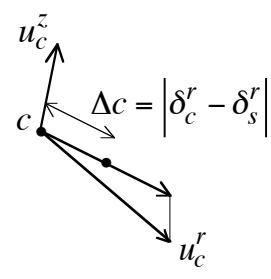

Fig. 6. Vector $u_{c}^{r}$ is projected onto the element surface to define the direction of the movement of $c$. If $\delta_{c}^{r}$ reaches $\delta_{s}^{r}, c$ slides by $\left|\delta_{c}^{r}-\delta_{s}^{r}\right|$. 
In IS4TM: International Symposium on Surgery Simulation and Soft Tissue Modelling Lecture Notes in Computer Science (LNCS 2673), N. Ayache and H. Delingette (Eds), Springer Verlag, New York. 2003. pp. 311-320.

\section{Construction of the Pre-calculated Responses}

The pre-calculated force-deflections $f_{l, m}^{z}\left(\delta^{z}\right)$ and $f_{l, m}^{r}\left(\delta^{r}\right)$, as well as the friction properties described by $\mu\left(\delta^{z}\right)$, can be interpolated by piecewise linear or polynomial interpolators. The parameters of the interpolators are calculated such that the outputs of the haptic system fit either the actual forces obtained by experimental measurement or accurate forces generated by an offline simulator [14, 9].

The location and number of testing points can differ from those of the surface nodes used to represent a virtual body. For example, Figure 7 illustrates how a mesh can be refined by obtaining the force response at a new node from precalculated forces at three test points, using an interpolation approach similar to the one of the last section. More generally, the number of the test points for a body can be much smaller than the number of virtual body surface nodes used for a simulation. This is useful when it is known that the change of force response over the surface of a body is smoother than, for example, the change in visual aspect or in the geometry of the body surface.

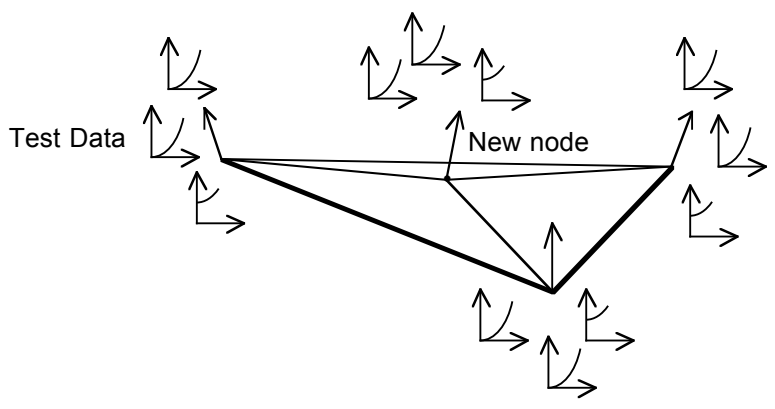

Fig. 7. Force Deflection responses for a new node of a mesh of a body surface is obtained by force-deflection responses of three test points using area interpolation.

\section{Implementation}

A simple haptic engine system was implemented to evaluate the performance of the interpolation approach. The virtual environment comprised a tool interacting with a deformable cylindrical virtual body made of about 500 patches. The local responses at a node $l$ of element $m$ were:

$$
\begin{aligned}
& f_{l, m}^{z}\left(\delta^{z}\right)= \begin{cases}0 & \delta^{z} \leq 0 \\
100 k_{l, m}^{z} \delta^{z^{2}} & 0<\delta^{z}\end{cases} \\
& f_{l, m}^{r}\left(\delta^{r}\right)=k_{l, m}^{r} \delta^{r}
\end{aligned}
$$

The vectors $u_{l, m}^{z}$ at each mode were in the radial direction. 


\section{In IS4TM: International Symposium on Surgery Simulation and Soft Tissue Modelling Lecture Notes in Computer Science (LNCS 2673), N. Ayache and H. Delingette (Eds), Springer Verlag, New York. 2003. pp. 311-320.}

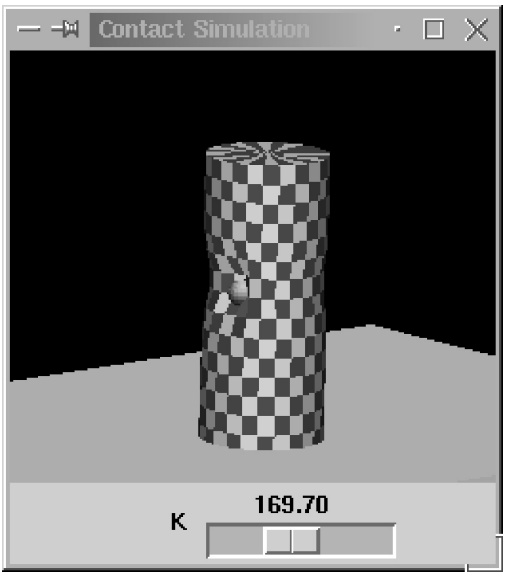

Fig. 8. Graphical user interface and device used in the tool contact simulation.

The test showed that coordinates interpolation, together with force-deflection interpolation provided continuity for the responses. Conversely, eliminating one of the two interpolations caused a variety of artifacts, including unwanted textures or limit cycles resulting from lack of passivity during sliding motions. These could be attributed to discontinuities in deflection or in the produced force at the mesh edges. These unwanted artifacts were called haptic 'clicks' and 'pops' by analogy to the defects in sound production.

Figure 8 shows the user interface used in the test. The simulation program consisted of two independent real-time threads running under RTLinux-3. One thread provided for rendering the forces and the other for finding the active element. The forces were generated by a PenCat/Pro ${ }^{\mathrm{TM}}$ haptic device (Immersion Canada Inc.) which is "direct drive" and hence provides good fidelity because of near absence of mechanical damping.

\section{Conclusion}

This paper described a pre-calculation solution for haptic simulation of contact between a tool and a nonlinear deformable body. The approach relies on pre-calculated forces responses known at nodes of the triangulated body surface mesh. It calculates forces at arbitrary locations through interpolation of both force responses and coordinates. According to the needs and the application, piecewise linear or polynomial interpolators can be used to register normal and lateral forces at each node at each direction for a range of deflection distances. The pre-calculation responses can be derived from actual force responses resulted from measurement or accurate offline simulations. The measurements or simulations can be done only at a set of testing point located over the surface of the body and the results are extended to many nodes of the body surface by using area interpolation. 


\section{In IS4TM: International Symposium on Surgery Simulation and Soft Tissue Modelling Lecture Notes in Computer Science (LNCS 2673), N. Ayache and H. Delingette (Eds), Springer Verlag, New York. 2003. pp. 311-320.}

This approach could be generalized to cases that include damage and plasticity by adding additional internal variables. The case of cutting, a special case of damage, was treated in [13]. The systems approach also allows us to study stability of haptic interactions in a general way [15].

\section{Acknowledgement}

This research was funded by the project "Reality-based Modeling and Simulation of Physical Systems in Virtual Environments" of IRIS, the Institute for Robotics and Intelligent Systems (Canada's Network of Centers of Excellence). Additional funding is provided by NSERC, the Natural Sciences and Engineering Council of Canada, in the form of an operating grant for the second author.

\section{References}

1. Armstrong-Hélouvry, B., Dupont, P., and Canudas De Wit., C. 1994. A Survey of Models, Analysis Tools and Compensation Methods for the Control of Machines with Friction. Automatica, 30(7):1083-1138

2. Astley, O. R., Hayward, V. 1998. Multirate Haptic Simulation Achieved by Coupling Finite Element Meshes Through Norton Equivalents. Proc. IEEE International Conference on Robotics and Automation, pp. 989-994.

3. Astley, O., Hayward V. 2000. Design Constraints for Haptic Surgery Simulation. Proc. IEEE International Conference on Robotics and Automation, pp. 2446-2451.

4. Cotin, S., Delingette, H., Ayache, N. 1999. Real-time Elastic Deformations of Soft Tissues for Surgery Simulation. IEEE Transactions on Visualization and Computer Graphics, Vol. 5:1, pp. 62-73.

5. D'Aulignac, D., Balaniuk, R., Laugier, C. 2000. A Haptic Interface for a Virtual Exam of the Human Thigh. Proc. IEEE International Conference on Robotics and Automation, pp. 2452-2457.

6. Debunne, G., Desbrun, M., Cani, M., Barr, A. 2001. Dynamic Real-Time Deformations Using Space and Time Adaptive Sampling. Computer Graphics and Interactive Techniques, SIGGRAPH 2001, ACM Press, pp. 31-36.

7. Delingette, H., Cotin, S., Ayache, N. 1999. A Hybrid Elastic Model Allowing Realtime Cutting, Deformations and Force-feedback for Surgery Training and Simulation. Computer Animation Proceedings, pp. 70-81.

8. Frank, A. O., Twombly, A. I., Barth, T. J., Smith, J. D. 2001. Finite Element Methods for Real-Time Haptic Feedback of Soft-Tissue Models in Virtual Reality Simulators. Proc. of the Virtual Reality Conference, pp. 257-263.

9. Greenish, S., Hayward, V., Chial, V., Okamura, A., Steffen, T. 2002. Measurement, Analysis and Display of Haptic Signals During Surgical Cutting. Presence: Teleoperators and Virtual Environments, MIT Press. Vol. 6(11). pp. 626-651.

10. Hayward, V., Armstrong, B. 2000. A New Computational Model of Friction Applied to Haptic Rendering. In Experimental Robotics VI, P. I. Corke and J. Trevelyan (Eds.), Lecture Notes in Control and Information Sciences, Vol. 250, Springer-Verlag, pp. 403-412.

11. James, D. L., Pai, D. K. 1999. ArtDefo, Accurate Real Time Deformable Objects. SIGGRAPH 99 Conference Proceedings, pp. 65-72. 
In IS4TM: International Symposium on Surgery Simulation and Soft Tissue Modelling Lecture Notes in Computer Science (LNCS 2673),

N. Ayache and H. Delingette (Eds), Springer Verlag, New York. 2003. pp. 311-320.

12. James, D. L., Pai D. K. 2001. A Unified Treatment of Elastostatic and Rigid Contact Simulation for Real Time Haptics. Haptics-e, the Electronic Journal of Haptics Research, Vol. 2, No. 1.

13. Mahvash, M., Hayward, V. 2000. Haptic Rendering of Cutting, A Fracture Mechanics Approach. Haptics-e, the Electronic Journal of Haptics Research, Vol. 2, No. 3.

14. Mahvash, M., Hayward, V., Lloyd, J. E. 2002. Haptic Rendering of Tool Contact. Proc. Eurohaptics 2002. pp. 110-115.

15. Mahvash, M., Hayward, 2003. Passivity-Based High-Fidelity Haptic Rendering of Contact. 2003. Proc. IEEE International Conference on Robotics and Automation, in print.

16. Bro-Nielsen, M. 1998. Finite Element Modeling in Surgery Simulation. Proceedings of the IEEE, 86:3, pp. 490-503.

17. Wu, X., Downes, M. S., Goktekin, T., Tendick, F. 2001. Adaptive Nonlinear Finite Elements for Deformable Body Simulation Using Dynamic Progressive Meshes. Proc. Eurographics 2001, Vol. 20, No. 3, pp. 349-58.

18. Zhuang, Y., Canny, J. 2000. Haptic Interaction with Global Deformations. Proc. IEEE International Conference on Robotics and Automation, pp. 2428-2433. 